\title{
VALIDEZ Y CONFIABILIDAD DEL TEST DE IDENTIFICACIÓN DE LOS TRASTORNOS DEBIDOS AL CONSUMO DE ALCOHOL (AUDIT) EN ESTUDIANTES DE UNA UNIVERSIDAD CHILENA ${ }^{1}$
}

\author{
VALIDITY AND RELIABILITY OF THE ALCOHOL USE \\ DISORDERS IDENTIFICATION TEST (AUDIT) IN A CHILEAN \\ UNIVERSITY STUDENTS
}

\author{
Fredy Seguel Palma* \\ Gema Santander ManRíQuez ${ }^{* *}$ \\ Omar AleXandre Barriga ${ }^{* * *}$
}

\begin{abstract}
RESUMEN
Objetivo: analizar la confiabilidad y validez del test de Identificación de los Trastornos Debidos al Consumo de Alcohol en una población de estudiantes que ingresan por primera vez a la Universidad. Material y método: aplicación del instrumento a 845 estudiantes, previo consentimiento informado, seleccionados mediante muestreo aleatorio estratificado por facultad. 789 alumnos lo completaron satisfactoriamente, se aplicaron pruebas paramétricas para obtener resultados de consumo de alcohol basados en los puntajes de corte y zonas sugeridas por la Organización Mundial de la Salud. Se aplicó alfa de Cronbach para determinar la fiabilidad y análisis factorial para la validez. Resultados: La confiabilidad del instrumento fue apropiada ( $\alpha$ $=0,83)$, y se detectaron dos componentes referidos al consumo de alcohol y las consecuencias. Conclusión: este test es un instrumento confiable y válido para diagnóstico en el consumo de alcohol tanto riesgoso como dependiente en estudiantes universitarios.
\end{abstract}

Palabras clave: Alcoholismo, validez de las pruebas, confiabilidad, estudiantes, enfermería psiquiátrica.

\begin{abstract}
Objective: to analyze the reliability and validity of the Alcohol Use Disorders Identification Test in a population of students who are entering the university for their first time. Material and method: application of the instrument to 845 students, previous informed consent, selected through faculty stratified random sampling. 789 students completed it successfully, parametric tests for alcohol consumption were applied to obtain results based on cut scores and areas suggested by the World Health Organization. Cronbach's alpha was applied to determine the reliability and factor analysis for validity. Results: The reliability of the instrument was appropriate $(\alpha=0.83)$, and detected two components relating to alcohol consumption
\end{abstract}

\footnotetext{
${ }^{1}$ Publicación derivada del proyecto de investigación titulado: "Magnitud del consumo de drogas lícitas e ilícitas de los estudiantes que ingresan por primera vez a la Universidad Austral de Chile, aprobado por la Dirección de Investigación y Desarrollo de la Universidad Austral de Chile, 2008, Valdivia, Chile.

*Enfermero. Docente Instituto de Enfermería, Facultad de Medicina, Universidad Austral de Chile. E-mail: fredyseguel@ uach.cl

${ }^{* *}$ Enfermera. Docente Universidad Mayor. E-mail: gema.santander@umayor.cl

${ }^{* * *}$ Licenciado en Relaciones Internacionales. Docente Departamento de Sociología, Universidad de Concepción -Chile.

E-mail: obarriga@udec.cl
} 
and consequences. Conclusion: This test is a reliable and valid diagnosis tool in both hazardous alcohol consumption and dependency in college students.

Key words: Alcoholism, validity of tests, reliability, students, psychiatric nursing.

Fecha recepción: 27/03/12 Fecha aceptación: 07/03/13

\section{INTRODUCCIÓN}

En América Latina los estudiantes universitarios presentan prevalencias altas de consumo de alcohol en relación a la población general, sobrepasando el $60 \%$ (1), con un consumo importante durante la vida y también en los últimos 12 meses (2), este comportamiento es calificado como endémico por la gran magnitud del problema y su impacto en salud pública(2-4). Es el principal problema de salud en los centros educacionales de nivel superior, en diferentes países, lo que ha resultado en una preocupación permanente para los gobiernos en desarrollar políticas que permitan abordarlo; por otro lado, las universidades son consideradas, en países como los Estados Unidos, lugares de alto riesgo de consumo por presentar, incluso, prevalencias superiores a la población de la misma edad que no estudia en la universidad y por mostrar estrecha relación con la producción de muertes, lesiones, agresiones físicas y sexuales entre los estudiantes (5). El consumo excesivo de alcohol en estudiantes universitarios puede causar gran impacto en el rendimiento académico al dedicar menor cantidad de horas al estudio e incluso limitando las capacidades neurocognitivas (6).

En Chile el consumo de alcohol en la educación superior alcanza un 29,6\%, marcando una tendencia a la disminución a partir del año 2006. Según datos del Servicio Nacional para la Prevención y Rehabilitación del Consumo de Drogas y Alcohol (SENDA) (7), la mayor tasa de abuso en la población general se encuentra en las edades comprendidas entre 19 y 25 años, lo que demuestra que los estudiantes de educación superior presentan mayor riesgo de sufrir consecuencias negativas al pertenecer a este rango etario, por ello es necesario que existan instrumentos validados en Chile no sólo en población general sino que específicamente en las poblaciones vulnerables.

Para la detección del consumo de riesgo, perjudicial y dependencia se ha utilizado el Test de Identificación de los trastornos debido al consumo de alcohol (AUDIT, por sus siglas en inglés), este instrumento fue desarrollado bajo la tutela de la Organización Mundial de la Salud (OMS) para identificar riesgos de uso abusivo del alcohol, evidenciando gran ventaja en la detección del peligro incluso para aquellas personas que no presentan dependencia. Este instrumento originalmente es dividido en tres subescalas que miden el consumo del alcohol, las conductas derivadas del consumo y las consecuencias de éste; sin embargo, otros estudios factoriales realizados posteriormente evidencian dos subescalas: las primeras tres preguntas referidas al consumo de alcohol y las siete preguntas restantes relacionadas con las consecuencias negativas experimentadas cuando se bebe o simplemente una escala con unidimensionalidad $(8,9)$.

Puede ser aplicado en distintas áreas de la comunidad para la detección del uso problemático del alcohol, tales como: clínicas de atención primaria, servicios de urgencias, consulta médica, sala de hospital, policlínico ambulatorio, hospital psiquiátrico, tribunal, comisaría, prisión, ejército y centros laborales (10).

Existen escasos estudios (11) de validación del instrumento en población de estudiantes 
universitarios, solamente se evidenciaron 3; la investigación efectuada en Nigeria a 810 estudiantes universitarios (12), la realizada en los Estados Unidos a 391 estudiantes universitarios (13) y la realizada en Colombia a 459 estudiantes universitarios (14), de éstos sólo el último presentó análisis factorial del instrumento. Estas investigaciones demostraron que el AUDIT es un cuestionario válido para la detección de problemas relativos al alcohol en esta población.

Se encontraron mayor cantidad de estudios de validación en poblaciones específicas no universitarias y general; en México se validó en 80 pacientes esquizofrénicos, de ambos sexos, mayores de 16 años, de la consulta externa del Instituto Nacional de Psiquiatría de la ciudad de México, en donde el test AUDIT mostró una consistencia interna alta ( $\alpha$ de Cronbach=0,812) (15). En Grecia fue probada su confiabilidad y validez en 218 sujetos con edad promedio de 40,7 años, de los cuales 109 fueron diagnosticados como dependientes del alcohol de acuerdo con el Diagnostic and Statistical Manual of Mental Disorders (DSM-IV), 4a edición y 109 sujetos fueron considerados como controles saludables. La confiabilidad interna fue de 0,8 tanto para los controles como para las personas dependientes del alcohol, con medias significativamente más bajas para el primer grupo ( $\mathrm{p}<0,001)(16)$. En hombres casados senegaleses se estudió a 62 individuos con bajo riesgo en el consumo de alcohol y 88 con consumo dependiente o peligroso, determinados mediante el Quantity Frequency Questionary y el Alcohol Use Module of the Composite International Diagnostic Interview (CIDI), mostrando que este test puede ser utilizado satisfactoriamente para diagnosticar el consumo (17). En Washington se evaluó la validez concurrente del test en comparación con: el DSM-IV, la escala de dependencia al alcohol, el inventario de consecuencias del bebedor, la escala del bebedor obsesivo compulsivo, la escala de percepciones sobre la abstinencia, el porcentaje de transferrin carbohidrato deficiente y la gama glutamil transferasa, concluyendo que es un instrumento pertinente no sólo como escala para detección a nivel de la población general sino que también para detectar la severidad de la dependencia al alcohol (18). Es necesario destacar que el test AUDIT fue validado en población general en Chile en el estudio realizado por Alvarado et al., en el año 2009, cuyo análisis factorial evidenció las tres subescalas propuestas originalmente: consumo de alcohol, síntomas de dependencia y consecuencias negativas del consumo (19).

Contar con escalas validadas, y específicamente este instrumento, permite al profesional de enfermería utilizar una herramienta práctica y objetiva para el abordaje de una población vulnerable en cuanto al consumo de alcohol, que acude escasamente a los centros asistenciales y que no percibe la magnitud del problema e impacto en la familia y sociedad, por lo tanto, la enfermera constituiría un agente de promoción de la salud al trabajar con las universidades y prevenir las consecuencias al detectar a tiempo el consumo problemático.

El objetivo de este estudio es analizar la confiabilidad y validez del test AUDIT en una población de estudiantes que ingresan por primera vez a la Universidad Austral de Chile.

\section{MATERIAL Y MÉTODO}

\section{Descripción del instrumento}

El AUDIT está constituido por 10 ítemes, basados en los criterios del CIE-10 para detectar a los pacientes con síntomas de dependencia, consumo nocivo de alcohol y consumo peligroso (20), la respuesta sólo toma breves minutos y el máximo puntaje corresponde a 40 puntos. Originalmente fue diseñado de manera de integrar preguntas que abarquen los ámbitos conceptuales del consumo de alco- 
hol, la conducta de consumo (dependencia) y los problemas relacionados con el alcohol, con el objetivo de realizar un diagnóstico de riesgo en la comunidad, siendo herramienta útil para el equipo de salud de atención primaria, por lo tanto se puede dividir en tres subescalas: las tres primeras preguntas referidas al consumo de alcohol, las siguientes 3 a los síntomas de dependencia y las últimas dos a las consecuencias negativas del consumo $(21,22)$.

Respecto de la clasificación en base al puntaje, la OMS recomienda cuatro zonas, las que se asocian a intervenciones que se pueden realizar con los individuos de acuerdo al riesgo, la zona I (< 8 pts.) corresponde al bajo riesgo o abstinencia donde solamente es necesaria una educación referente al alcohol; la Zona II ( 8 - 15 pts.) hace referencia al consumo por sobre el bajo riesgo recomendando el consejo simple, la zona III (16 - 19 pts.) se aborda como consumo de riesgo y perjudicial mediante consejo simple, terapia breve y monitorización continua, y la zona IV (20 - 40 pts.), posible dependencia al alcohol, existe derivación al especialista para evaluación diagnóstica y tratamiento (10).

\section{Aplicación del instrumento y análisis estadístico}

El AUDIT fue autoaplicado a una muestra probabilística comprendida por 845 alumnos de primer año, estratificada por campus y facultad, incluyó 3 campus, dentro de los cuales abarcó 8 facultades con un total de 33 carreras. El porcentaje de respuestas completas del AUDIT para el cálculo de la puntuación alcanzó un 93,4\% (789 estudiantes), los cuales respondieron positivamente frente al consumo en los últimos 12 meses. Para el análisis de las subescalas y los puntajes se tomaron en cuenta tres subescalas y cuatro zonas descritas por la OMS y por Alvarado et al. (19), contrastando los resultados obtenidos mediante pruebas paramétricas. En el análisis de fiabilidad y validez interna se aplicó el alfa de Cronbach aceptando valores superiores a 0,7 (aceptable) y 0,8 (ideal). Se analizaron las correlaciones total-elemento para determinar la importancia de cada pregunta en el instrumento y la robustez de su estructura obteniendo la validez de constructo. Cumpliendo con los supuestos del análisis factorial variables continuas y tamaño muestral, se aplicó la medida de adecuación muestral de Kaiser-Meyer-Olkin y la prueba de esfericidad de Bartlett para comprobar la correcta utilización del análisis para este instrumento y población, con valores aceptables superior a 0,5 y menor a 0,05 correspondientemente. Se analizaron las correlaciones de Pearson estableciendo correspondencias que permitieron determinar a simple vista algunas agrupaciones de preguntas. Mediante el método de componentes principales, en las comunalidades, se analizó la contribución de las preguntas a la variabilidad del instrumento y se aplicó técnicas estadísticas de rotación Várimax y Oblimin para determinar los componentes resultantes. A través de la varianza total explicada se determinaron los componentes adecuados con autovalores iniciales superiores a 1,0 y dentro de la matriz de componentes se identificó las preguntas que pertenecían a las dimensiones correspondientes.

\section{Aspectos éticos de la investigación}

El estudio fue aprobado por el Comité de Ética de la Facultad de Medicina de la Universidad Austral de Chile. Los estudiantes respondieron voluntariamente luego de la firma del consentimiento informado. La aplicación del instrumento en terreno fue desarrollada por estudiantes de enfermería de los últimos años de la carrera capacitados. La encuesta fue anónima, sin embargo los estudiantes que presentaban inquietudes podían anotar el Rol Único Tributario (RUT) para identificarlos y establecer contacto. 


\section{RESULTADOS}

La población en estudio comprendió a 745 estudiantes, los cuales correspondían en un $51,8 \%$ al sexo masculino, con un promedio de edad de 19,2 años (IC 19,05 - 19,27; DS $=1,5)$. El promedio de puntaje en la escala general fue de $4,9(\mathrm{IC}=4,6-5,3 ; \mathrm{DS}=5.0)$ y la mediana de 4 puntos. De acuerdo con lo establecido por la OMS para las puntuaciones del TEST según zonas, en esta población de estudio resultó un $76,7 \%$ con bajo riesgo o abstinencia (zona I, media $=2,7$ pts., DS=2,3 pts.), $19,5 \%$ con consumo sobre el bajo riesgo (zona II, media $=10,8$ pts., $D S=2,3$ ), $1,6 \%$ consumo perjudicial (zona III, media $=17,2$ pts., $\mathrm{DS}=1,1$ pts.) y $2,2 \%$ posible dependencia (Zona IV, media $=22,6$ pts., DS $=3,7$ pts.), siendo estas diferencias estadísticamente significativas $(\mathrm{p}=0,000)$. Por otra parte, al basarse en las recomendaciones de las tres subescalas de la OMS, el análisis de fiabilidad por cada una de ellas para esta población sólo es aceptable para la escala 1 (preguntas $1,2,3)$ (Tabla 1).

Tabla 1: Alfa de Cronbach según test total y subescalas.

\begin{tabular}{lc}
\hline Escala & Alfa de Cronbach \\
\hline Total AUDIT & 0,828 \\
Sub escala 1 (preguntas $1,2,3)$ & 0,802 \\
Sub escala 2 (preguntas $4,5,6)$ & 0,658 \\
Sub escala 3 (preguntas $7,8,9)$ & 0,615 \\
\hline
\end{tabular}

El análisis de confiabilidad mostró un alfa de Cronbach ( $\alpha$ ) de 0,828 con un total de población de 707 estudiantes de primer año. $\mathrm{Al}$ analizar el estadístico total-elemento se muestra una correlación elemento-total corregida por sobre $0,4(0,41-0,68)$ en las preguntas 1 a 9 . La pregunta 10 presenta un valor de 0,34 .

Por otro lado, al analizar el alfa de
Cronbach si se elimina el elemento, las preguntas 1 a 8 muestran una contribución al instrumento importante haciendo referencia al consumo de la persona y las consecuencias que pudiera acarrear para ella $(\alpha=$ $0,79-0,82)$, mientras que la pregunta 9 se encuentra al límite $(\alpha=0,83)$ y la pregunta 10 presenta una leve mejoría $(\alpha=0,834)(\mathrm{Ta}-$ bla 2). Estas dos preguntas apuntan a lo que

Tabla 2: Estadísticos Total-Elemento test AUDIT.

\begin{tabular}{lccc}
\hline \multicolumn{1}{c}{ Preguntas } & $\begin{array}{c}\text { Correlación } \\
\text { elemento-total } \\
\text { corregida }\end{array}$ & $\begin{array}{c}\text { Correlación } \\
\text { múltiple al } \\
\text { cuadrado }\end{array}$ & $\begin{array}{c}\text { Alfa de } \\
\text { Cronbach si } \\
\text { se elimina el } \\
\text { elemento }\end{array}$ \\
\hline $\begin{array}{l}\text { 1. ¿Con qué frecuencia se toma alguna bebida que } \\
\text { contenga alcohol? }\end{array}$ & 0,565 & 0,353 & 0,807 \\
$\begin{array}{l}\text { 2. ¿Cuántas bebidas que contengan alcohol se toma } \\
\text { normalmente cuando bebe? }\end{array}$ & 0,599 & 0,522 & 0,803 \\
$\begin{array}{l}\text { 3. ¿Con qué frecuencia se toma 6 o más bebidas } \\
\text { que contengan alcohol en un solo día? }\end{array}$ & 0,677 & 0,59 & 0,794 \\
\hline
\end{tabular}


4. ¿Con qué frecuencia, en el curso del último año, ha sido incapaz de parar de beber una vez que había empezado?

5. ¿Con qué frecuencia, en el curso del último año, no pudo hacer lo que esperaba de usted porque había bebido?

6. ¿Con qué frecuencia, en el curso del último año, ha necesitado beber en ayunas para recuperarse después de haber bebido mucho el día anterior?

7. ¿Con qué frecuencia, en el curso del último año, ha tenido remordimientos o sentimientos de culpa después de haber bebido?

8. ¿Con qué frecuencia, en el curso del último año, no ha podido recordar lo que sucedió la noche anterior porque había estado bebiendo?

9. Usted o alguna otra persona, ¿han resultado heridos porque usted había bebido?

10. ¿Algún familiar, amigo, médico o profesional sanitario han mostrado preocupación por su consumo de bebidas alcohólicas o le han sugerido que deje de beber?

$\begin{array}{ccc}0,555 & 0,338 & 0,809 \\ 0,534 & 0,337 & 0,813 \\ 0,502 & 0,299 & 0,817 \\ 0,5 & 0,31 & 0,814 \\ 0,614 & 0,43 & 0,804 \\ 0,414 & 0,198 & 0,825 \\ 0,335 & 0,132 & 0,834\end{array}$

rrelación entre las variables para realizar un análisis factorial.

La matriz de correlaciones muestra en general resultados bajo 0,3 para las preguntas 9 y 10 a excepción de la pregunta 9 en correlación con la pregunta 3,7 y 8 , por otro lado las tres primeras preguntas presentan correlaciones importantes entre sí y más bajas con el resto (Tabla 3). Esto evidencia que las interrogantes pueden apuntar por lo menos a

Tabla 3. Matriz de correlaciones interítem AUDIT.

\begin{tabular}{rlllllllllll}
\hline & & P1 & P2 & P3 & P4 & P5 & P6 & P7 & P8 & P9 & P10 \\
\hline & P1 & 1.000 & & & & & & & & & \\
& P2 & 0,48 & 1.000 & & & & & & & & \\
& P3 & 0,529 & 0,704 & 1.000 & & & & & & & \\
& P4 & 0,345 & 0,396 & 0,44 & 1.000 & & & & & & \\
& P5 & 0,299 & 0,351 & 0,423 & 0,426 & 1.000 & & & & & \\
& P6 & 0,302 & 0,291 & 0,377 & 0,375 & 0,431 & 1.000 & & & & \\
& P7 & 0,347 & 0,31 & 0,313 & 0,31 & 0,346 & 0,288 & 1.000 & & & \\
& P8 & 0,383 & 0,357 & 0,434 & 0,449 & 0,421 & 0,417 & 0,496 & 1.000 & & \\
P9 & 0,27 & 0,263 & 0,323 & 0,257 & 0,289 & 0,242 & 0,322 & 0,371 & 1.000 & \\
P10 & 0,251 & 0,22 & 0,223 & 0,259 & 0,19 & 0,268 & 0,222 & 0,259 & 0,148 & 1.000 \\
\hline
\end{tabular}


dos componentes en el instrumento.

En el análisis de componentes principales, las comunalidades previo a la rotación muestran menor ponderación en las preguntas 9 y 10 pues el grado de explicación que presenta de parte de los componentes es bajo (32,5 y $23,2 \%$ correspondientemente). Los componentes resultantes corresponden a 2 que presentan autovalores iniciales totales mayores que 1 con una retención del 51,6\% de la varianza. Todas las variables se correlacionan positivamente con el componente 1, en cambio para el componente 2 sólo las preguntas 2 y 3 en forma negativa. Al igual que los resultados anteriores con las rotaciones Varimax y Oblimin se formaron dos componentes que retienen igual porcentaje de variabilidad (51,6\%) (Tabla 4).

Tabla 4. Varianza Total Explicada análisis de componentes principales.

\begin{tabular}{lcccccc}
\hline Componente & $\begin{array}{c}\text { Autovalores } \\
\text { iniciales }\end{array}$ & $\begin{array}{c}\text { Sumas de las } \\
\text { saturaciones al } \\
\text { cuadrado de } \\
\text { la extracción }\end{array}$ & $\begin{array}{c}\text { Suma de las } \\
\text { saturaciones al } \\
\text { cuadrado de } \\
\text { la rotación } \\
\text { Várimax }\end{array}$ & $\begin{array}{c}\text { Suma de las } \\
\text { saturaciones } \\
\text { al cuadrado } \\
\text { de la rotación } \\
\text { Oblimin }\end{array}$ \\
\cline { 2 - 7 } & Total & $\begin{array}{c}\text { \% de la } \\
\text { varianza }\end{array}$ & Total & Total & $\begin{array}{c}\text { \% de la } \\
\text { varianza }\end{array}$ & Total \\
\hline 1 & 4,154 & 41,537 & 2,805 & 3,668 & 28,047 & 3,668 \\
2 & 1,003 & 10,028 & 2,352 & 3,228 & 23,517 & 3,228 \\
3 & 0,895 & & & & & \\
4 & 0,827 & & & & & \\
5 & 0,693 & & & & & \\
6 & 0,618 & & & & & \\
7 & 0,561 & & & & & \\
8 & 0,518 & & & & & \\
9 & 0,453 & & & & & \\
10 & 0,279 & & & & & \\
\hline
\end{tabular}

En estas dos últimas pruebas estadísticas las tres primeras preguntas se ponderan importantemente con el componente 2 mostrando valores comprendidos entre 0,690 y 0,866 ; por otro lado, las preguntas 4 a 10 presentan mayor correlación con el componente 1 mostrando ponderaciones que van desde 0,466 a 0,738 , donde la pregunta $\mathrm{N}^{\circ}$ 10 presenta el menor valor. Es necesario resaltar que en la rotación Oblimin se pueden observar otras correlaciones, sin embargo las más fuertes muestran el comportamiento antes mencionado. En este sentido se puede rescatar que para esta población podemos encontrar dos subcategorías en el AUDIT, la primera (componente 2) correspondiente al consumo propiamente tal (preguntas $1-3$ ) en cuanto a las frecuencias tanto en general como en el momento de consumo y día determinado, ésta presenta una puntuación total de 12 pts. con un promedio en la población estudiada de 3,1 pts. (IC= $2,9-3,2, \mathrm{DS}=$ 2,39 ). La segunda (componente 1 , preguntas 4 - 10) se relaciona con la detección de de- 
pendencia al alcohol, sintomatología como la incapacidad de parar de beber, dejar de hacer actividades debido a la bebida, culpabilidad, necesidad de beber en ayunas, problemas de memoria, violencia hacia los demás y preocupación de terceros por el comportamiento hacia la bebida (Tabla 5), presenta una puntuación máxima de 28 pts. El puntaje máximo obtenido en esta población fue de 20 pts., con un promedio de 1,9 pts. (IC $=1,7-2,1$, $\mathrm{DS}=2,8)$.

Tabla 5. Matriz de Componentes(a)

\begin{tabular}{|c|c|c|c|c|c|c|}
\hline \multirow[t]{2}{*}{ Preguntas } & \multicolumn{2}{|c|}{$\begin{array}{l}\text { Componente } \\
\text { Sin Rotación }\end{array}$} & \multicolumn{2}{|c|}{$\begin{array}{c}\text { Componente } \\
\text { Rotación Varimax }\end{array}$} & \multicolumn{2}{|c|}{$\begin{array}{c}\text { Componente } \\
\text { Rotación Oblimin }\end{array}$} \\
\hline & 1 & 2 & 1 & 2 & 1 & 2 \\
\hline $\begin{array}{l}\text { 1. ¿Con qué frecuencia se toma alguna bebida } \\
\text { que contenga alcohol? }\end{array}$ & 0,665 & & & 0,69 & 0,48 & $-0,741$ \\
\hline $\begin{array}{l}\text { 2. ¿Cuántas bebidas que contengan alcohol se } \\
\text { toma normalmente cuando bebe? }\end{array}$ & 0,703 & $-0,536$ & & 0,866 & 0,436 & $-0,882$ \\
\hline $\begin{array}{l}\text { 3. ¿Con qué frecuencia se toma } 6 \text { o más be- } \\
\text { bidas que contengan alcohol en un solo día? }\end{array}$ & 0,769 & $-0,451$ & & 0,844 & 0,53 & $-0,891$ \\
\hline $\begin{array}{l}\text { 4. ¿Con qué frecuencia, en el curso del último } \\
\text { año, ha sido incapaz de parar de beber una } \\
\text { vez que había empezado? }\end{array}$ & 0,672 & & 0,544 & & 0,639 & $-0,535$ \\
\hline $\begin{array}{l}\text { 5. ¿Con qué frecuencia, en el curso del último } \\
\text { año, no pudo hacer lo que esperaba de usted } \\
\text { porque había bebido? }\end{array}$ & 0,657 & & 0,622 & & 0,679 & $-0,449$ \\
\hline $\begin{array}{l}\text { 6. ¿Con qué frecuencia, en el curso del últi- } \\
\text { mo año, ha necesitado beber en ayunas para } \\
\text { recuperarse después de haber bebido mucho } \\
\text { el día anterior? }\end{array}$ & 0,618 & & 0,646 & & 0,676 & \\
\hline $\begin{array}{l}\text { 7. ¿Con qué frecuencia, en el curso del último } \\
\text { año, ha tenido remordimientos o sentimien- } \\
\text { tos de culpa después de haber bebido? }\end{array}$ & 0,611 & & 0,673 & & 0,689 & \\
\hline $\begin{array}{l}\text { 8. ¿Con qué frecuencia, en el curso del último } \\
\text { año, no ha podido recordar lo que sucedió la } \\
\text { noche anterior porque había estado bebien- } \\
\text { do? }\end{array}$ & 0,725 & & 0,738 & & 0,78 & $-0,452$ \\
\hline $\begin{array}{l}\text { 9. Usted o alguna otra persona, ¿han resulta- } \\
\text { do heridos porque usted había bebido? }\end{array}$ & 0,525 & & 0,543 & & 0,57 & \\
\hline $\begin{array}{l}\text { 10. ¿Algún familiar, amigo, médico o profe- } \\
\text { sional sanitario han mostrado preocupación } \\
\text { por su consumo de bebidas alcohólicas o le } \\
\text { han sugerido que deje de beber? }\end{array}$ & 0,431 & & 0,466 & & 0,48 & \\
\hline
\end{tabular}

(a) Método de extracción: Análisis de componentes principales. 2 componentes extraídos 
El grado de correlación entre los dos componentes con rotación Oblimin es de 0,56 . Al aplicar la prueba t-student, la diferencia de promedios entre los componentes es significativa $(p<0,000)$, los estudiantes universitarios de esta muestra evidencian un mayor consumo, pero menor grado de dependencia o consecuencias negativas en torno al alcohol. Al realizar la correlación entre el puntaje total del AUDIT y los dos componentes resultan significativas $(\mathrm{p}<0,000)$ con correlaciones importantes (componente $1=0,90$ y componente $2=0,86$ ); por lo tanto el test puede ser utilizado como una escala única o como dos escalas separadas.

Estos dos componentes presentan diferencias significativas en relación al sexo ( $p$ $<0,000$ ), siendo la puntuación promedio mayor para los hombres tanto en el componente 1 (Hombres, media $=2,2$; Mujeres, media $=1,4)$ como en el 2 (Hombres, media $=$ 3,4 ; Mujeres, media $=2,4$ ), por lo tanto, los varones muestran mayor consumo y consecuencias negativas en torno al alcohol.

$\mathrm{Al}$ analizar la confiabilidad y el comportamiento factorial de cada componente, el componente 1 presenta un alfa de Cronbach de 0,75 , aceptable. En general la correlación de las preguntas con éste, a excepción de la $\mathrm{N}^{\circ} 10$, es buena y el alfa de Cronbach al eliminar el elemento solo en esta pregunta produce un aumento que no es importante $(\alpha=0,76)$. En la medida KMO presentó un valor de 0,85 y una prueba de esfericidad de Bartlett significativa $(p=0,000)$, por lo tanto, se puede realizar un análisis factorial. En este análisis las preguntas se correlacionan bien con un solo componente que retiene el $43,2 \%$ de la variabilidad, con ponderaciones que van desde 0,48 (pregunta $\mathrm{N}^{\circ} 10$ ) a 0,78 (pregunta $\mathrm{N}^{\circ} 8$ ). No se puede realizar la prueba de componentes rotados.

El componente 2 obtuvo un alfa de Cronbach de 0,80 , lo que muestra una validez interna importante, por otro lado la prueba de Medidas de adecuación muestral de $\mathrm{KMO}(0,676)$ y la prueba de esferi- cidad de Bartlett $(\mathrm{p}=0,000)$ resultaron con valores aceptables para realizar una factorización. Según el análisis, la pregunta $\mathrm{N}^{\circ} 1$ no contribuye a este componente ya que al evaluar los estadísticos total elemento el alfa de Cronbach aumenta si éste se elimina (a $=0,82$ ), por otro lado, en las pruebas factoriales sólo se identifica la formación de un solo componente con el cual las preguntas se correlacionan muy bien, resultando ponderaciones que van desde $0,78-0,89$.

\section{DISCUSIÓN Y CONCLUSIÓN}

El promedio de puntuación concentrada en el bajo riesgo concuerda con los puntajes obtenidos en investigaciones realizadas en mujeres (23), estudiantes universitarios (24) y en estudiantes de enfermería (25) y dice relación con la frecuencia con que se presenta el fenómeno de consumo en forma decreciente desde el bajo consumo o bajo riesgo, con alta población, hasta la dependencia donde el número de sujetos es menor.

El instrumento AUDIT es breve y simple para responder (11), validado en Chile en población general (19) y a nivel internacional en ciertos grupos específicos relacionados con la atención en salud, sin embargo la contribución al conocimiento es insuficiente en estudiantes universitarios que presentan altas tasas de consumo poniendo en riesgo su futuro profesional y en última instancia el desarrollo del país, haciendo importante la necesidad de ver el comportamiento en cuanto a la validez y confiabilidad del instrumento de detección $(12,13)$.

Los resultados de este estudio muestran una confiabilidad importante para el instrumento AUDIT, lo que está en concordancia con investigaciones realizadas en adolescentes $(\alpha=0,83)(21)$, personas con desórdenes mentales $(\alpha=0,89)(26)$, en atención primaria $(\alpha=0,81)(27)$ y lo encontrado en revisiones de la literatura (28), donde se describe 
que es un buen instrumento para la detección no solo del riesgo sino que también de la dependencia en el consumo de alcohol. En ciertos estudios, algunas preguntas marcan diferencias en el comportamiento factorial al realizar el análisis de los estadísticos total elemento, como es el caso de la validación realizada en Grecia donde los alfas son más altos si se elimina el elemento en las preguntas $1 \mathrm{y}$ 9 (16) o la pregunta 4 en otras investigaciones (15). En este estudio las preguntas 9 y 10 presentan valores de correlación en el límite, sin embargo, no existe justificación estadística o teórica suficiente como para eliminarlas, pues en base a éstas y los resultados que arrojan las preguntas 7 y 8 permite discriminar el consumo perjudicial de alcohol de acuerdo a las recomendaciones de la OMS.

En esta población, las dimensiones o dominios sugeridos por la OMS para el instrumento no se ven reflejadas, puesto que en el análisis factorial se determinan solo dos componentes, que se relacionan con el consumo propiamente tal y con las consecuencias que acarrea un consumo excesivo y que tienen que ver con la dependencia, ya sea en sus inicios con los primeros síntomas como con síndromes avanzados, ratificado con los resultados obtenidos en el análisis de fiabilidad para cada subescala: esto se describe también en otros estudios que concuerdan con lo adecuado de este resultado (28), como el realizado por Gómez en población adolescente, quien determinó en su análisis factorial dos componentes con una explicación de la varianza de $41,62 \%$ para el primer componente al cual pertenecen las preguntas 4 a la 10 y de $11,46 \%$ para el segundo componente donde las preguntas 1, 2, 3 correlacionan en forma considerable (27) y lo establecido por Bergman en población general, donde dos factores explican el 55\% de la variabilidad de la prueba descartando las tres dimensiones en favor de lo adecuado del primer análisis (29). Sin embargo, otros estudios como el de Alvarado et al. (19) plantea una diferencia importante evidenciando tres dimensiones con alfas de Cronbach por sobre 0,8 en cada sub escala y AUDIT total. Por su parte Ospina determinó en población universitaria, la formación de una sola dimensión al correlacionar todos los ítemes con un solo componente y un valor propio de 3,54 (Eigenvalue), sugiriendo la unidimencionalidad del instrumento, sin embargo con una varianza explicada baja de 35,41\% (14). Lo mismo sucede con skipsey en pacientes que abusaron de sustancias, quien determinó que todas las preguntas correlacionaban muy bien con un solo factor (9).

A pesar de lo variado de estos análisis, el resultado final se centra en que el test AUDIT es un cuestionario confiable y válido para la detección de los diferentes niveles en el consumo de alcohol, práctico en su aplicación y fácil de analizar. Los resultados de este estudio avalan su utilización como instrumento íntegro para su aplicación en esta población específica. La diferencia importante está señalada por la formación de las distinas categorías que en este caso son dos: la frecuencia de consumo propiamente tal y las consecuencias del consumo excesivo involucrando, dentro de ello, el grado de dependencia y el riesgo. Esta escala es una buena herramienta para acudir a los lugares donde se concentra la población universitaria vulnerable y realizar la detección de las personas en riesgo, dependencia o daño debido al consumo abusivo de alcohol, para realizar las intervenciones correspondientes de prevención, mitigación o tratamiento oportuno, y paralelamente formular un diagnóstico y evaluación adecuados del comportamiento del consumo que sirva de información para la toma de decisiones a las autoridades universitarias.

\section{Implicancias del estudio para enfermería}

El profesional de enfermería trabaja activamente con la comunidad en programas de promoción de la salud y de prevención de la enfermedad en poblaciones vulnerables. En 
este caso la población universitaria constituiría un foco de atención para hacer la detección precoz de un problema tan trascendental para la sociedad debido a sus graves consecuencias, con una herramienta validada, fácil de aplicar y concreta en su interpretación. Esto resulta ser una oportunidad y a la vez un desafío para el/la enfermero/a en cuanto a la formulación de estrategias de abordaje que le permitan acudir a los diferentes centros de educación superior a establecer puentes de conexión con los jóvenes, para la instauración de programas de prevención en coordinación con las autoridades universitarias, pues los estudiantes solo se acercan al centro de salud cuando se sienten enfermos y el beber en exceso, en esta etapa, se considera como algo natural debido a la falta de conciencia en lo vulnerable del ser humano cuando ha consumido alcohol.

\section{REFERENCIAS}

1. Piedra K, O’Brien B, Pillon S. Drugs use and risk behavior in a university community. Rev Lat Am Enfermagem. 2005; 13(Número Especial): 1194-200.

2. Guerra A, Vieira P, García L. I Levantamento Nacional Sobre o Uso de Álcool, Tabaco y Otras Drogas entre Universitarios de 27 Capitales Brasileiras. Brasilia: SENAD; 2010.

3. Do Santos A, Pereira R, Soares N, Alvaci $\mathrm{D}$, Carim $\mathrm{K}$ et al. Use of psychoactive drugs by health sciences undergraduate students at the Federal University in Amazonas. Cad Saude Publica. 2006; 22(3): 663-71.

4. Consejo Nacional para el Control de Estupefacientes (CONACE), Ministerio del Interior. Octavo estudio nacional de drogas en población general de Chile 2008. Gobierno de Chile (Santiago): Ministerio del Interior; 2008 Junio. 22 p.

5. Díaz A, Díaz L, Hernández C, Narro J,
Fernández $\mathrm{H}$ et al. Prevalencia del consumo riesgoso y dañino de alcohol y factores de riesgo en estudiantes universitarios de primer ingreso. Salud Mental. 2008; 31(4): 271-82.

6. Mota N, Alvarez R, Corral M, Rodríguez $\mathrm{S}$, Parada $\mathrm{M}$ et al. Risky alcohol use and heavy episodic drinking among spanish university students: a two-year followup. Gac Sanit. 2010; 24(5): 372-77.

7. Servicio Nacional para la Prevención y Rehabilitación del Consumo de Drogas y Alcohol, SENDA. Noveno estudio nacional de drogas en población general de Chile, 2010. Observatorio Chileno de Drogas (Santiago): Ministerio del Interior y Seguridad Pública; 2011. 34 p.

8. Babor TF, Higgins-Biddle JC, Saunders JB, Monteiro MG. AUDIT Cuestionario de Identificación de los Transtornos debidos al Consumo de Alcohol. Pautas para su utilización en Atención Primaria. Ginebra, Suiza: Organización Mundial de la Salud Departamento de Salud Mental y Dependencia de Sustancias - Generalitat Valenciana Conselleria de Benestar Social; 2001.40 p.

9. Meneses C, Zuardi A, Loureiro S, Crippa J. Alcohol Use Disorders Identification Test (AUDIT): an updated systematic review of psychometric properties. Psychol Neurosci. 2009; 2(1): 83-97.

10. Adewuya AO. Validation of the alcohol use disorders identification test (audit) as a screening tool for alcohol-related problems among Nigerian university students. Alcohol Alcohol. 2005; 40(6): 57577.

11. Kokotailo PK, Egan J, Gangnon R, Brown $\mathrm{D}$, Mundt $\mathrm{M}$ et al. Validity of the alcohol use disorders identification test in college students. Alcohol Clin Exp Res. 2004; 28(6): 914-20.

12. Villamil SV, Valencia CM, Medina-Mora Icaza ME, Juárez GF. Validation of the alcohol use disorders identification test (AUDIT) in Mexican patients with schi- 
zophrenia. Rev Panam Salud Pública. 2009; 26(4): 283-89.

13. Moussas G, Dadouti G, Douzenis A, Poulis E, Tzelembis A et al. The Alcohol Use Disorders Identification Test (AUDIT): reliability and validity of the Greek version. Ann Gen Psychiatry. 2009; 8(11): 1 -5.

14. De SP, Jayawardana P, Pathmeswaran A. Concurrent validity of the alcohol use disorders identification test (AUDIT). Alcohol Alcohol. 2008 Jan; 43(1): 49-50.

15. Donovan DM, Kivlahan DR, Doyle SR, Longabaugh R, Greenfield SF. Concurrent validity of the Alcohol Use Disorders Identification Test (AUDIT) and AUDIT zones in defining levels of severity among out-patients with alcohol dependence in the COMBINE study. Addiction. 2006; 101(12): 1696-704.

16. Santis R, Garmendia ML, Acuña G, Alvarado $\mathrm{ME}$, Arteaga O. The Alcohol Use Disorders Identification Test (AUDIT) as a screening instrument for adolescents. Drug Alcohol Depend. 2009; 103(3): 155-58.

17. Volk RJ, Steinbauer JR, Cantor SB, Holzer CE. The Alcohol Use Disorders Identification Test (AUDIT) as a screen for at-risk drinking in primary care patients of different racial/ethnic backgrounds. Addiction. 1997; 92(2): 197-206.

18. Saunders JB, Aasland OG, Babor TF, de la Fuente JR, Grant M. Development of the Alcohol Use Disorders Identification Test (AUDIT): WHO Collaborative Project on Early Detection of Persons with Harmful Alcohol Consumption--II. Addiction. 1993; 88(6): 791-804.

19. Alvarado ME, Garmendia ML, Acuña G, Santis R, Arteaga O. Assessment of the alcohol use disorders identification test (AUDIT) to detect problem drinkers. Rev Med Chil. 2009; 137(11): 1463-468.

20. Aalto M, Alho H, Halme JT, Seppa K. The alcohol use disorders identification test
(AUDIT) and its derivatives in screening for heavy drinking among the elderly. Int J Geriatr Psychiatry. 2010; (9): 881-85.

21. Perula de Torres LA, Fernández-García JA, Arias-Vega R, Muriel-Palomino M, Márquez-Rebollo E et al. Validation of the AUDIT test for identifying risk consumption and alcohol use disorders in women. Aten Primaria. 2005; 36(9): 499506.

22. Arrieta K. Consumo patológico de alcohol entre los estudiantes de la Universidad de Cartagena, 2008. Rev Salud Publica. $2009 ; 11(6)$ : 878-86.

23. Matute RC, Pillon SC. Alcohol consumption by nursing students in Honduras. Rev Lat Am Enfermagem. 2008; 16(Spec No): 584-89.

24. Durbeej N, Berman A, Gumper C, Palmstiema T, Krisstiensson M, Alm C. Validation of the Alcohol Use Disorders Identification Test and the Drug Use Disorders Identification Test in a Swedish sample of suspected offenders with signs of mental health problems: Results from the Mental Disorder, Substance Abuse and Crime study. J Subst Abuse Treat. 2010; 39(4): 364-77.

25. Gómez AA, Conde MA, Alberto Aguiar BJ, Manuel Santana MJ, Jorrin MA et al. Diagnostic usefulness of Alcohol Use Disorders Identification Test (AUDIT) for detecting hazardous alcohol consumption in primary care settings. Med Clin. 2001; 116(4): 121-24.

26. Reinert DF, Allen JP. The Alcohol Use Disorders Identification Test (AUDIT): a review of recent research. Alcohol Clin Exp Res. 2002; 26(2): 272-79.

27. Karno M, Granholm E, Lin A. Factor structure of the Alcohol Use Disorders Identification Test (AUDIT) in a mental health clinic sample. J Stud Alcohol. 2000; 61(5): 751-58.

28. Maisto SA, Conigliaro J, McNeil M, Kraemer K, Kelley ME. An empirical investi- 
gation of the factor structure of the AUDIT. Psychol Assess. 2000; 12(3): 346-53. 29. Skipsey K, Burleson JA, Kranzler HR. Utility of the AUDIT for identification of hazardous or harmful drinking in drugdependent patients. Drug Alcohol Depend. 1997; 45(3): 157-63. 